\title{
Potential of thalidomide and thalidomide analogues as immunomodulatory drugs in leprosy and leprosy reactions
}

\author{
G. KAPLAN \\ Laboratory of Cellular Physiology \& Immunology, The Rockefeller \\ University, New York, New York, USA
}

Erythema nodosum leprosum (ENL), a serious complication of lepromatous leprosy, typically includes painful subcutaneous nodules accompanied by systemic symptoms of fever, malaise, wasting, and most importantly, peripheral neuropathy. Since Sheshkin reported, ${ }^{1}$ in 1965 , that thalidomide alleviates both the nodules and the systemic symptoms, this drug has been used to treat ENL. However, the mechanism of action of thalidomide in ENL remained unknown until recently.

In collaboration with Dr E. Sampaio and Dr E. Sarno, the effects of thalidomide on leukocyte function in ENL patients were examined. ${ }^{2}$ In these studies, monocytes isolated from normal individuals were stimulated in vitro with lipopolysaccharide (LPS) or mycobacterial products, in the presence or absence of thalidomide, and the production of cytokine by the cells was evaluated. At physiological levels, $1-10 \mu \mathrm{g}$ per ml, the drug was shown to inhibit partially the production of tumour necrosis factor- $\alpha(\mathrm{TNF} \alpha)$, without significantly reducing the production of other monocyte cytokines. In further studies in vitro, it was observed that thalidomide reduces the half-life of the mRNA for TNF $\alpha$ from about 30 to $17 \mathrm{~min}^{3}$

These results were subsequently extended to clinical studies. Patients with active ENL were found to have elevated serum levels of TNF $\alpha$. Treatment with thalidomide, $300 \mathrm{mg}$ daily for the first week, $200 \mathrm{mg}$ daily for the second week, and $100 \mathrm{mg}$ daily for the third week, rapidly reduced the serum levels of $\mathrm{TNF} \alpha$, in association with improvement of clinical symptoms. ${ }^{4}$ Histopathologically, the characteristic mixed inflammatory infiltrate in the dermis, the vasculitis and the keratinocyte activation, evidenced by expression of HLA-DR and ICAM, were improved following treatment with thalidomide. In addition, treatment of ENL patients with thalidomide was shown to reduce the capacity of the patients' peripheral blood mononuclear cells (PBMC) to release $\mathrm{TNF} \alpha$ in response to stimulation in vitro. Taken together, these studies suggested that the improvement of ENL following treatment with thalidomide was associated with the reduction in $\mathrm{TNF} \alpha$ levels.

The ability of thalidomide to inhibit production of TNF $\alpha$ in ENL patients suggested that the drug may be beneficial in other diseases, in the pathogenesis of which TNF $\alpha$ plays a role, including other mycobacterial infections, tuberculosis and Mycobacterium avium infection, and HIV infection. In one study, Tramontana et al. ${ }^{5}$ treated 30 tuberculosis patients with thalidomide, in a dosage of $300 \mathrm{mg}$ per day, or placebo for one or more 14-day cycles. Thalidomide, which was well tolerated by these patients, resulted in significant reduction of serum TNF $\alpha$ levels. In addition, PBMC isolated from these patients at various intervals and stimulated with LPS showed a reduction in $\mathrm{TNF} \alpha$ production only when the patients were 
under treatment with thalidomide. Interestingly, during treatment with thalidomide, patient serum interferon- $\gamma(\mathrm{IFN} \gamma)$ levels increased slightly. Compared to placebo-treated patients, those treated with thalidomide showed accelerated weight gain during treatment. Further studies showed that, among patients dually infected with $M$. tuberculosis and HIV, serum TNF $\alpha$ levels were initially higher than among those infected with HIV only, suggesting that the opportunistic mycobacterial infection stimulated production of $\mathrm{TNF} \alpha{ }^{6}$ In addition, plasma TNF $\alpha$ and HIV-1 levels were correlated. When patients with tuberculosis and HIV were treated with thalidomide, there was a reduction of plasma levels of both TNF $\alpha$ and HIV-1, accompanied by significant weight gain. Among patients with HIV infection only, treatment with thalidomide did not result in significant change of either TNF $\alpha$ or HIV-1 levels, although there was some weight gain in this group. In vitro, thalidomide was shown to inhibit production of virus in the latently HIV-infected monocytoid cell-line U1. ${ }^{7}$

Treatment of patients who had both tuberculosis and HIV infection with thalidomide was shown to increase plasma IL-12 levels, ${ }^{8}$ as well as increased levels of soluble IL-2 receptor and soluble CD8. Patients treated with placebo showed no such effect. In addition, the proliferative response in vitro to mycobacterial antigens (PPD) of these dually infected patients' PBMC was increased following thalidomide treatment. In this study, no effect of the drug on viral load was observed. In patients infected with HIV only, treatment with thalidomide resulted in similarly increased plasma levels of IL-12, soluble IL-2 receptor and soluble CD8. ${ }^{9}$

Similar immune stimulation may be of importance in other chronic inflammatory diseases of non-infectious origin. Recently, we have shown that thalidomide treatment of patients with scleroderma results in increased plasma IL-12 levels and clinical improvement, evidenced by decreased skin fibrosis and healing of digital ulcers. ${ }^{9}$ When PBMC of scleroderma patients were exposed in vitro to anti-CD3 antibody in the presence of thalidomide, there was increased production of IL-2, IL-3, GM-CSF and IFN $\gamma$, suggesting that thalidomide had exerted a co-stimulatory effect and induced activation of T-cells.

In HIV-infected individuals, thalidomide-induced immune activation was associated with a significant increase of body weight (reversal of wasting). When HIV patients were maintained on a strict, isocaloric diet during thalidomide treatment, the weight gain was shown to result from an increase of lean body mass. ${ }^{10}$ Again, no effect on viral load was noted. Recently, a large multicentre, randomized, placebo-controlled study of the effect of thalidomide on the wasting associated with HIV was completed. ${ }^{11}$ Significant weight gain was observed among patients who were treated with thalidomide in a dosage of 100 or $200 \mathrm{mg}$ per day for 8 weeks. The drug was well tolerated, although some mild-to-moderate rashes were noted during therapy.

The use of thalidomide in clinical settings may be limited by its associated toxic manifestations, including teratogenicity, peripheral neuropathy, gastrointestinal disturbances, rash, fever and drowsiness. ${ }^{12}$ Therefore, it was important to develop similar drugs that exhibited analogous immunomodulatory activities without the thalidomide-associated toxicity. For this purpose, a thalidomide-analogue programme was begun with Celgene Corporation (Warren, NJ, USA) for development of novel immunomodulatory compounds. Initially, compounds were synthesized and selected for enhanced inhibition of TNF $\alpha$ in vitro in PBMC stimulated by LPS. ${ }^{13,14}$ In addition to inhibition of $\mathrm{TNF} \alpha$, these compounds enhanced production of IL-10. A selected series of structural analogues of thalidomide was found to inhibit production of TNF $\alpha$ significantly; the $50 \%$ inhibitory concentration $\left(\mathrm{IC}_{50}\right)$ ranged from $280 \mu \mathrm{M}$ to $0.02 \mu \mathrm{M}$. The drugs that showed enhanced activity in vitro were then 
tested in vivo, and shown to inhibit production of TNF $\alpha$ in the murine LPS-induced shock model. ${ }^{13,15}$ Furthermore, one of these analogues was shown to block development of adjuvant arthritis in the rat. ${ }^{16}$ This analogue also inhibited production of TNF $\alpha$ in rat splenocytes in vitro.

Upon further characterization, a number of the analogues were found to segregate into two distinct families. ${ }^{17}$ One group was found to inhibit production of TNF $\alpha$ efficiently, did not inhibit monocyte IL-6, and stimulated production of IL- 8 and IL-10. These analogues also inhibited phosphodiesterase-IV (PDE-IV). The second group of thalidomide analogues inhibited the production of TNF $\alpha$, IL- 6 and IL-8, and also strongly stimulated production of IL-10; this second group of molecules did not inhibit PDE-IV. ${ }^{17,18}$

The two groups of thalidomide analogues were also tested for their effects on T-cells. ${ }^{17,19}$ As might be expected from earlier studies of inhibitors of PDE-IV, those analogues that inhibited PDE-IV showed slightly reduced T-cell activation, as measured by thymidine incorporation, as well as reduced production of the T-cell cytokines IL- 2 and IFN $\gamma$. By contrast, those analogues that did not inhibit PDE-IV stimulated T-cell activation, causing increased lymphocyte proliferation and increased production of IL- 2 and IFN $\gamma$. Thalidomide, the parent compound, behaved like the compounds that did not inhibit PDE-IV, and was also shown to stimulate T-cell activation in vitro. Thus, thalidomide and the other compounds that did not inhibit PDE-IV appeared to inhibit monocyte activation, while stimulating T-cell activation. Most strikingly, this class of compounds inhibited monocyte TNF $\alpha$ production, whereas T-cell production of $\mathrm{TNF} \alpha$ was unaffected or even slightly increased. Moreover, monocyte-dependent production of IL-12 was inhibited, whereas these drugs enhanced Tcell-dependent production of IL-12. The stimulatory effect on T cells was more pronounced in the CD8+ subset of $\mathrm{T}$ cells.

These exciting results suggest that thalidomide and its analogues could be used more extensively in leprosy reactions. Identification of a non-teratogenic analogue of thalidomide for treatment of ENL is critically important, because ENL remains a major complication of leprosy. Indeed, even years after completion of multidrug therapy for leprosy, ENL may still occur. Many of the patients at risk of developing ENL are young women of childbearing age, for whom thalidomide treatment is contraindicated because of its teratogenic effects.

Thalidomide has not been successful in the treatment of reversal reactions, perhaps because $\mathrm{T}$-cell activation appears to be a significant component of reversal reactions, and thalidomide has been shown to stimulate T-cell activation. However, the analogues of thalidomide, which inhibit PDE-IV and block production of TNF $\alpha$ but do not stimulate T cells, may be candidate drugs for the treatment of reversal reactions.

\section{References}

I Sheskin J. Thalidomide in the treatment of lepra reactions. Clin Pharmacol Therap, 1965; 6: 303-306.

2 Sampaio EP, Samo EN, Gallily R et al. Thalidomide selectively inhibits TNF $\alpha$ production by stimulated human monocytes. J Exp Med, 1991; 173: 699-703.

3 Moreira AL, Sampaio EP, Zmuidzinas A et al. Thalidomide exerts its inhibitory action on tumor necrosis factor- $\alpha$ by enhancing mRNA degradation. J Exp Med, 1993; 177: 1675-1680.

4 Sampaio EP, Kaplan G, Miranda A et al. The influence of thalidomide on the clinical and immunologic manifestation of erythema nodosum leprosum. J Infect Dis, 1993; 168: 408-414.

5 Tramontana JM, Utaipat U, Molloy A et al. Thalidomide treatment reduces TNF- $\alpha$ production and enhances weight gain in patients with pulmonary tuberculosis. Molec Med, 1995; 1: 384-397. 
${ }^{6}$ Klausner JD, Makonkawkeyoon S, Akarasewi P et al. The effect of thalidomide on the pathogenesis of human immunodeficiency virus type 1 and $M$. tuberculosis infection. $J A I D S, 1996 ; 11: 247-257$.

${ }^{7}$ Makonkawkeyoon S, Limson-Pobre RNR, Moreira AL et al. Thalidomide inhibits the replication of human immunodeficiency virus type-1. Proc Natl Acad Sci USA, 1993; 90: 5974-5978.

8 Bekker L-G, Haslett PAJ, Maartens G et al. Thalidomide-induced antigen-specific immune stimulation in patients with HIV-1 and tuberculosis. J Infect Dis, 2000; 181: 954-965.

9 Oliver SJ, Moreira A, Kaplan K. Immune stimulation in scleroderma patients treated with thalidomide. Clin Immun, 2000; in press.

${ }^{10}$ Haslett P, Hempstead M, Seidman C et al. The metabolic and immunologic effects of short-term thalidomide treatment of patients infected with the human immunodeficiency virus. AIDS Res Human Retroviruses, 1997; 13: 1047-1054.

11 Kaplan G, Thomas S, Fierer DS et al. Thalidomide for the treatment of patients with AIDS-associated wasting. AIDS Res Human Retroviruses, 2000; in press.

12 Haslett $\mathrm{P}$, Tramontana J, Burroughs $\mathrm{M}$ et al. Adverse reactions to thalidomide in patients infected with the human immunodeficiency virus. Clin Infect Dis, 1997; 24: 1223-1227.

13 Corral LG, Muller GW, Moreira AL et al. Selection of novel analogues of thalidomide with enhanced TNF- $\alpha$ inhibitory activity. Molec Med, 1996; 2: 506-515.

14 Muller GW, Corral LG, Shire MG et al. Structural modifications of thalidomide produce analogs with enhanced TNF inhibitory activity. J Med Chem, 1996; 39: 3238-3240.

15 Moreira AL, Wang J, Sarno EN, Kaplan G. Thalidomide protects against LPS-induced shock. Brazilian J Med Biol, 1997; 30: 1199-1207.

16 Oliver SJ, Freeman SL, Kaplan G. Thalidomide analog CC1069 inhibits development of rat adjuvant arthritis. Clin Exp Immunol, 1999; 118: 315-321.

17 Corral LG, Haslett PAJ, Muller GW et al. Differential cytokine modulation and T cell activation by two distinct classes of thalidomide analogs which are potent inhibitors of TNF- $\alpha$. J Immunol, 1999; 163: 380-386.

18 Muller GW, Chen R, Huang S-Y et al. Amino-substituted thalidomide analogues: potent inhibitors of TNF- $\alpha$ production. Biorg Med Chem Ltt, 1999; 9: 1625-1630.

19 Haslett PAJ, Corral LG, Albert M, Kaplan G. Thalidomide costimulates primary human T lymphocytes, preferentially inducing proliferation, cytokine production and cytotoxic responses in the CD8+ subset. $J$ Exp Med, 1988; 187: 1885-1892.

\section{DISCUSSION}

Dr Ottenhoff: Did you test whether the effect of thalidomide is dependent upon IFN $\gamma$ ?

Dr Kaplan: We believe that IL-12 up-regulates CD4 T-ligand, which up-regulates T-cell activation, which activates secretion of IFN $\gamma$, yielding a feedback loop. It is all independent upon IL-2.

Dr Gillis: Are some patients with ENL refractory to treatment by thalidomide?

Dr Kaplan: Some patients simply don't respond very well to thalidomide. More common, however, is the situation in which patients respond, but the ENL recurs when treatment is stopped. Finally, some patients, once treated by thalidomide, do not experience recurrence of ENL. We are beginning to think that thalidomide affects other cytokines in addition to TNF $\alpha$, and have undertaken a study with Dr Roche in Nepal to investigate this matter further. 